\title{
PROMOÇÃO DA SAÚDE MATERNA A PARTIR DO VIVIDO DO PARTO DE MULHERES CARDIOPATAS*
}

\author{
Thaís Vasconselos Amorim¹ Ívis Emília de Oliveira Souza², Anna Maria de Oliveira Salimena ${ }^{1}$, Ana Luiza de \\ Oliveira Carvalho ${ }^{2}$, Lúcia de Fátima da Silva ${ }^{3}$, Tassiane Ferreira Langendorf ${ }^{4}$
}

\begin{abstract}
RESUMO: Objetivou compreender os significados do processo parturitivo a partir da vivência do risco gestacional da mulher portadora de cardiopatia. Estudo qualitativo de abordagem fenomenológica, com coleta de dados entre julho e dezembro de 2014, em hospital para alto risco materno na Região Sudeste do Brasil. Dezessete participantes foram entrevistadas e os significados expressos analisados na perspectiva teórico-metodológica de Martin Heidegger. As mulheres significaram o medo de morrer no parto; e a preocupação com a sobrevivência do bebê, pedindo ao médico para dar prioridade em salvá-lo. Evidenciou-se o desvelamento do temor frente ao medo expresso, implicando em vulnerabilidade que cardiopatas vivenciam ao engravidarem. Isto porque, para além das possíveis complicações advindas da doença cardíaca, residem subjetividades que são apartadas do processo de cuidado em saúde, especialmente no que diz respeito à falta de diálogo e tomada de decisão unilateral acerca do tipo, momento e via de parto.
\end{abstract}

DESCRITORES: Saúde materna; Cardiopatias; Gravidez de alto risco; Parto; Enfermagem.

\section{MATERNAL HEALTH PROMOTION BASED ON THE BIRTH EXPERIENCE OF WOMEN WITH HEART DISEASE}

\begin{abstract}
The aim of this study was to understand the meanings of the birth process based on the experiences of high-risk pregnancies of women with heart disease. A qualitative study with a phenomenological approach, with data collection happening between July and December 2014, was developed at a high-risk pregnancy hospital in the Southeastern region of Brazil. Seventeen participants were interviewed and the expressed meanings were analyzed with the theoretical-methodological approach of Martin Heidegger. Women mentioned fearing death during birth; and worries about their infants' survival, asking physicians to prioritize their lives. The display of concerns facing the expressed fears stood out, involving vulnerabilities which patients with heart disease experience when pregnant. That is because, in addition to possible complications stemming from heart disease, there are subjective issues that are ignored during the healthcare process, especially related to lack of communication and one-sided decision making regarding the type, time and route of delivery.
\end{abstract}

DESCRIPTORS: Maternal health; Heart diseases; High-risk pregnancy; Delivery; Nursing.

\section{PROMOCIÓN DE SALUD MATERNA A PARTIR DE VIVENCIA DEL PARTO DE MUJERES CON CARDIOPATÍA}

RESUMEN: Se objetivó comprender los significados del proceso de parición partiendo de la vivencia del riesgo gestacional de mujeres con cardiopatía. Estudio cualitativo de abordaje fenomenológico. Datos recolectados de julio a diciembre de 2014 en hospital de alto riesgo materno de Región Sudeste de Brasil. Fueron entrevistadas diecisiete participantes. Los significados manifestados fueron analizados según perspectiva teórico-metodológica de Martin Heidegger. Las mujeres significaron el miedo a morir en el parto; y su preocupación por la supervivencia del bebé, pidiendo al médico priorizar su salvación. Se evidenció el desvelo del temor ante el miedo expresado, implicando en la vulnerabilidad que experimentan las mujeres con cardiopatía al quedar embarazadas. Más allá de las posibles complicaciones devenidas de la enfermedad cardíaca, residen subjetividades aparte del proceso de cuidado en salud, particularmente en lo que respecta a la falta de diálogo y toma unilateral de decisiones sobre el tipo, momento y vía de parto. DESCRIPTORES: Salud Materna; Cardiopatías; Embarazo de Alto Riesgo; Parto; Enfermería.

*Artigo extraído da tese intitulada: "Gestar Sendo-portadora-de-cardiopatia: contribuições para o cuidado em Saúde da Mulher". Universidade Federal do Rio de Janeiro, 2015.

${ }^{1}$ Enfermeira. Doutora em Enfermagem. Docente de Enfermagem da Universidade Federal de Juiz de Fora. Juiz de Fora, MG, Brasil.

${ }^{2}$ Enfermeira. Doutora em Enfermagem. Docente de Enfermagem da Universidade Federal do Rio de Janeiro. Rio de Janeiro, RJ, Brasil.

${ }^{3}$ Enfermeira. Doutora em Enfermagem. Docente de Enfermagem da Universidade Estadual do Ceará. Fortaleza, CE, Brasil. ${ }^{4}$ Enfermeira. Pós-doutoranda em Enfermagem. Universidade Federal de Santa Maria. Santa Maria, RS, Brasil.

Autor Correspondente:

Thaís Vasconselos Amorim

Universidade Federal de Juiz de Fora

R. Paulo de Souza Freire, 56 - 36025-350 - Juiz de Fora, MG, Brasil

E-mail: thaisamorim80@gmail.com
Recebido: 07/04/2017

Finalizado: $26 / 10 / 2017$ 


\section{INTRODUÇÃO}

A promoção da saúde materna se fortalece por meio de ações de cuidado que incluem o Planejamento Familiar e a assistência pré-natal, ambos na perspectiva do empoderamento e protagonismo da mulher no planejamento da gestação e parição, conforme apregoam programas e políticas vigentes ${ }^{(1-2)}$. Sabese ainda que a qualidade assistencial no pré-natal é diretamente proporcional à saúde materna e fetal, sendo imprescindíveis o acesso, as condições estruturais básicas e o adequado preparo dos profissionais nas diversas situações clínicas e obstétricas que podem se apresentar durante gestação, parto, nascimento e puerpério.

No contexto atual, é impositivo considerar a meta do terceiro Objetivo de Desenvolvimento Sustentável de redução global da razão de mortalidade materna (RMM) para 70 mortes a cada 100.000 nascidos vivos, para os próximos 15 anos. O Brasil possui como meta para 2030 reduzir a RMM para $20^{(3)}$, o que se constitui desafio frente ao aumento das doenças crônico degenerativas como as cardiopatias ${ }^{(4)}$.

O manejo clínico da doença cardíaca prévia ou da que se apresenta ao longo da gestação deve contemplar o planejamento de intervenções em todo o ciclo gravídico puerperal, com especial enfoque na prevenção de complicações que impliquem em morte materna evitável. Sabe-se que o alto risco gestacional associa-se a resultados maternos e neonatais desfavoráveis, imprimindo ainda mais atenção e necessidade de promoção à saúde da díade mãe-bebê ao longo do ciclo gravídico puerperal. Destaca-se a prevalência de óbitos nos períodos intraparto e pós-parto, considerando-se a relação entre eventos clínicos do período imediato ao nascimento e os ocorridos durante o trabalho de parto e parto ${ }^{(5-6)}$.

É recomendado que a avaliação da via de parto se faça em detrimento da cardiopatia, uma vez que a sobrecarga cardíaca é semelhante tanto no parto normal quanto no cesáreo. Todavia, os riscos inerentes à cesariana tais como infecções e outras complicações tornam o parto normal assistido mais seguro. Em ambos os casos, porém, deve-se monitorar cuidadosamente a díade mãe-filho, controlar o volume de líquidos infundidos e atentar-se para as alterações hemodinâmicas previstas como o aumento da pré-carga, da pressão arterial média e da perda sanguínea ${ }^{(4-7)}$.

Atreladas às questões fisiológicas e objetivas inerentes ao parto em mulheres portadoras de cardiopatia, encontram-se as de cunho subjetivo que, se apartadas da assistência, podem contribuir para a elevação da morbimortalidade, uma vez que a ansiedade pode imprimir aumento de até $60 \%$ do débito cardíaco, ocorrendo o mesmo durante as contrações uterinas ${ }^{(7)}$. Além da ansiedade, sentimentos como medo, estresse, impotência e culpa estão frequentemente relacionados às mulheres em condição de risco no ciclo gravídico puerperal, pela implicância desfavorável que a cardiopatia pode imprimir à saúde materna e dos bebês, anunciando a vulnerabilidade a que estão sujeitas ${ }^{(8-10)}$.

Tal paradigma se anuncia paradoxal, pois embora as condutas e protocolos médicos já estejam bem estabelecidos e resguardem desde a classificação do risco gestacional até o parto e acompanhamento após o nascimento, em instituição de referência para o risco materno, a mulher se sente insegura, temerosa e impotente, denotando o velamento da ciência à dimensão subjetiva ${ }^{(11)}$.

Desta forma, ouvir a mulher e compreender como vivenciou o parto na condição de risco por doença cardíaca possibilita à equipe de saúde, na qual o enfermeiro se insere ao perscrutar individual e socialmente o ser† em situação vulnerável, análise crítica e problematizadora da aderência de sua prática, em resposta às políticas e programas governamentais.

As pesquisas de enfermagem que orientam a prática profissional nãotem consideradoa especificidade cardiológica na gravidez de alto risco, sendo a produção científica incipiente e abordada do ponto de vista quantitativo. Frente a esta lacuna e pela relevância do cuidado em saúde que transcende os aspectos biológicos, sem direção à integralidade como princípio norteador da assistência, tornou-se objetivo compreender os significados do processo parturitivo a partir da vivência do risco gestacional da mulher portadora de cardiopatia.

† As palavras grafadas em itálico referem-se àquelas contidas no referencial de Martin Heidegger 
Estudo de natureza qualitativa, sustentado na abordagem fenomenológica de Martin Heidegger ${ }^{(12)}$. De modo a alcançar o objetivo proposto, a fenomenologia Heideggeriana se anunciou como método apropriado por permitir a objetividade das subjetividades, com o rigor científico de redução dos pressupostos, para que o sentido do ser possa ser desvelado.

Foram investigadas mulheres que gestaram na condição de risco por doença cardíaca. A etapa de coleta de dados ocorreu entre os meses de julho e dezembro de 2014, em instituição hospitalar de referência para alto risco materno na Região Sudeste do Brasil.

As fontes de dados se constituíram a partir dos registros em fichas de atendimento pré-natal das gestantes acompanhadas no cenário de estudo e que atenderam aos critérios de inclusão de serem portadoras de cardiopatia e vivenciarem ou já ter vivenciado a gestação sob risco, realizando o acompanhamento pré-natal na referida instituição. Foram critérios de exclusão ser menor de 18 anos e portadora de agravos mentais.

Posteriormente à busca dos registros e confirmada a possível inclusão no estudo, foi realizado contato telefônico com as mulheres, explicitando sobre a pesquisa e seu objetivo. Obteve-se consentimento por meio de assinatura do Termo de Consentimento Livre e Esclarecido de 17 participantes. A técnica de coleta de dados foi a entrevista aberta, na própria instituição, sendo somente uma em domicílio. Mediante roteiro de entrevista, direcionaram-se às mulheres questões orientadoras que versavam sobre o objeto em estudo.

As entrevistas duraram em média 32 minutos e foram gravadas em equipamento MP3. Utilizou-se ainda o diário de campo para registro dos conteúdos não verbais explicitados pelas participantes, quais gestos, choro, silêncio. Após a transcrição das entrevistas, procedeu-se a análise dos dados.

O referencial de Martin Heidegger ${ }^{(12)}$ se fundamenta no movimento analítico hermenêutico em duas etapas metódicas que se iniciam na escuta e leituras atentivas das entrevistas, buscando estruturas essenciais responsivas ao objetivo investigado. A aproximação destas estruturas ou trechos constituintes do conteúdo das entrevistas permitiu o alcance das Unidades de Significação como categorias de análise.

O primeiro momento analítico, denominado de compreensão vaga e mediana, se desdobrou a partir da compreensão ôntica ou dos fatos para as mulheres que gestaram sendo portadoras de cardiopatia. Mediante esta primeira compreensão, tornou-se possível a interpretação/hermenêutica dos sentidos na instância ontológica ou fenomenal, segundo o pensamento Heideggeriano. Destacase que a discussão deste artigo apresenta a hermenêutica alcançada em articulação com resultados de pesquisa da temática investigada.

O anonimato das participantes foi garantido mediante a utilização de códigos alfanuméricos simbolizados pela letra " $\mathrm{P}$ " e seguidos do número correspondente à ocorrência das entrevistas (P1, P2, P3...P17).

A pesquisa foi aprovada pelos Comitês de Ética em Pesquisa da Escola de Enfermagem Anna Nery da Universidade Federal do Rio de Janeiro e do Hospital Universitário Pedro Ernesto, sob os pareceres número 1.103.165 e 1.139.507, de junho de 2015.

\section{RESULTADOS}

A média de idade das 17 participantes foi de 30 anos e os diagnósticos de cardiopatia prévios à gestação distribuíram-se em cardiopatias por lesões isquêmicas, quatro (23\%), congênitas, uma (6\%), reumáticas, seis (36\%), distúrbios relacionados ao sistema de condução elétrica, duas (12\%) e lesões valvares, quatro $(23 \%)$.

O número total de gestações correspondeu a 40, sendo que 13 (76\%) não planejaram a gestação mais recente, quatro $(23 \%)$ mulheres tiveram uma gravidez, seis $(36 \%)$ duas gestações, quatro $(23 \%)$ tiveram 
três gestações e três (17\%) engravidaram quatro vezes. Relativo ao número de partos, cinco mulheres pariram uma única vez, oito tiveram dois partos e quatro pariram três vezes. Das 17 participantes, seis sofreram aborto em gestações prévias. As vias de parto se distribuíram ao longo de suas histórias reprodutivas em 19 (58\%) partos cesáreos e 14 (42\%) partos normais.

O movimento analítico hermenêutico permitiu a construção de duas Unidades de Significação. Deste modo, para as mulheres que participaram deste estudo, o vivido do parto significou: Terem sentido medo de morrer no parto; e Ficarem preocupadas com o bebê e pedirem ao médico para dar prioridade em salvá-lo.

Em terem sentido medo de morrer, as participantes referiram medo da morte por serem cardiopatas, listando os motivos de tal sentimento: medo de sofrerem parada cardíaca; de terem hemorragia; por ouvirem dizer que a pressão sobe; por saberem que corriam o risco de ter que fazer cesariana, conforme denotam os trechos constituintes das entrevistas selecionados a seguir:

[...] por causa do problema que eu tenho, que eu sou cardiopata, aí eu fiquei até com medo de eu ter ataque cardíaco na hora, uma parada cardíaca [...] porque diz que a pressão sobe, tanto que a minha subiu. (P1)

[...] eu estava com medo, eu estou lá fazendo força, o coração dispara, eu não tenho força nenhuma e aí como é que eles vão tirar a criança? (P2)

[...] o medo mesmo é só de morrer na hora do parto. Eu tenho medo de ter uma hemorragia na hora, o coração não suportar, parar de bater [...]. (P3)

[...] na hora do parto dela eu fiquei com muito medo de morrer, tive medo da pressão aumentar, de dar hemorragia. (P4)

Expressaram ainda seus medos relativos à via de parto e que por já terem passado por partos anteriores com a cardiopatia, acharam que pudesse acontecer coisas piores, conforme demonstram os trechos destacados:

[...] eu já tenho esse problema, vai ser a minha primeira cesárea. Como eu nunca tive uma cesárea estou bem preocupada de tanto ouvir o que as pessoas falam, eu estou medo da anestesia. (P5)

Dela eu pensava muito na hora do parto, eu só pensava que poderia acontecer de repente coisas ruins, é o medo com tudo que já passei antes. (P10)

[...] ontem quando eu internei, eu já fiquei agitada, coração aceleradíssimo, não dormi direito, eu acho assim que é uma ansiedade, então isso tudo já é uma questão de quem tem cardiopatia que já vai provocando esses sintomas. (P17)

Em ficarem preocupadas com o bebê e pedirem ao médico para dar prioridade em salvá-lo, as mulheres iniciaram suas compreensões significando medo de que algo acontecesse com seus filhos durante o parto por causa da cardiopatia. Consideraram que, se os médicos tivessem que dar preferência, deveriam salvar a vida do bebê. Declararam que disseram isto para os profissionais, embora soubessem que elas eram prioridades para estes. Os textos a seguir expressam esta compreensão:

[...] meu medo era essa taquicardia, em algum momento meu coração disparar, faltar oxigênio para ele e eles darem preferência, tipo salva a mãe ou o bebê entendeu? [...] essa médica veio me falar no exame: a prioridade aqui é você. Eu falei: mas eu não quero que eu seja a prioridade, eu quero que a prioridade seja meu filho, porque eu estou aqui por causa dele. (P2)

[...] Eu falei para médica: se acontece alguma coisa comigo na hora do parto, salva a minha filha, me deixa aí que eu já fiz o que tinha que fazer. (P4)

[...] Medo do meu bebê nascer e não resistir [...] O momento que eu senti mais medo foi quando o médico foi conversar comigo e com o meu marido dizendo que ele ia ter que fazer o parto de qualquer jeito [...] o risco era perder eu e meu filho de uma só vez. (P12)

Referiram ainda preocupação dos bebês nascerem prematuros e com problemas cardíacos pelas questões hereditárias. Dentre os relatos que demonstram esses significados: 
[...] vai que essa criança resolve nascer de sete meses? Porque a menina que estava acompanhando comigo ela já teve agora, a minha prima teve agora um bebê prematuro, tá internada, fiquei morrendo de medo, aí eu adiantei tudo. (P3)

[...] Minha filha é prematura. Por causa de eu ser cardiopata, eu tinha muito medo de perdê-la, que era o meu medo mesmo e nascer com algum problema, que foi o caso, que ela veio com quase as mesmas coisas que eu. (P9)

[...] Ah passa, passa sim, porque a gente sabe que tem as questões, como eu posso dizer, hereditárias. Então a gente fica com essa preocupação. (P17)

\section{- DISCUSSÃO}

No movimento existencial das participantes deste estudo, na interpretação do referencial teórico adotado, o medo desvela-se na dimensão ontológica como temor. Tais possibilidades são denominadas em suas variações como pavor, horror e terror. A distinção para constituição destes modos é interpretada em face da proximidade da ameaça e do caráter súbito em que essa ocorre.

Deste modo, o medo se torna pavor em função da situação que se apresenta como ameaça ser conhecida ou familiar, e chegar de modo súbito para o ser-aí-mulher. Para as participantes, a classificação da gravidez de risco já era algo familiar, pois possuíam o vivido de outras gestações também de risco. Assim, o risco de vida configurou-se como ameaça, transformando o medo em pavor.

Ao aproximar-se do momento do parto, o ser-aí-mulher compreende que seu medo aumentou, sustentando a interpretação do risco de vida implicado pela classificação do risco gestacional, o qual a ciência objetiva denota aumentar no parto pelas possíveis complicações hemodinâmicas de origem cardiológica tanto para a mãe quanto para o bebê $\hat{~}^{(7-8)}$. Em paralelo, ao retomar o medo significado pelas participantes, em seu caráter de aproximação e não familiaridade, este se transforma em horror, uma vez que o ser-aí-mulher desconhece como será o parto pelo prisma da gestação atual e em que condições o filho nascerá.

Pode ainda o que ameaça ter o súbito e a familiaridade do pavor conjugados com o não familiar do horror e, em se revelando ao mesmo tempo, o medo transformar-se existencialmente em terror ${ }^{(12)}$. Ao experimentar o pavor e o horror na ameaça de morte materna e fetal, o medo tornou-se terror, pois o risco pavoroso para si e seu filho e o desconhecimento que envolvia o parto horroroso poderiam transformar-se em morte terrorosa.

De modo semelhante aos resultados encontrados neste estudo, em gestantes hipertensas e portadoras de morbidade materna grave, a angústia e o medo da morte estiveram associados ao ciclo gravídico puerperal na perspectiva da prematuridade, condição clínica que também pode estar implicada pela doença cardíaca materna ${ }^{(8,13-14)}$. Neste caso, a prevenção do parto prematuro requer repouso e estabilização clínica da gestante, especialmente em mulheres com classe funcional I e II. A programação do parto se dará pelas condições maternas e fetais, podendo ter indicação de parto prétermo terapêutico quando da ocorrência de refratariedade clínica ao tratamento ${ }^{(10)}$.

Em paralelo, a decisão quanto ao momento mais adequado do nascimento, tipo de parto, anestesia e manejo da assistência perinatal em gestantes com cardiopatia congênita deve ser discutida com participação ativa de cardiologistas, obstetras, neonatologistas e anestesistas. E, em se tratando de classificações da capacidade funcional do coração em III e IV, há a recomendação de internação no terceiro trimestre de gestação com o intuito de planejar o parto, estabilizar e monitorar mãe e bebê, além de ajustar doses medicamentosas ${ }^{(10)}$.

Os resultados nesta pesquisa apontaram para um maior número de partos cesarianos, corroborando com dados de coorte que, embora não tenha encontrado diferenças estatísticas significativas entre intercorrências obstétricas e tipo de parto, observaram aumento de 3,44 vezes de chance de resultado neonatal desfavorável. O próprio aumento do risco gestacional, por si só, determinou 3,8 vezes mais possibilidades de resultados adversos maternos e 17,5 vezes maiores chances de pior resultado neonatal ${ }^{(6)}$. 
As malformações cardíacas fetais podem ou não estar associadas à cardiopatia materna. Frequentemente, os riscos aumentados de desenvolvimento de doenças cardíacas no feto estão relacionados à presença de cardiopatia congênita nos genitores, sendo mais incidentes quando da presença de doença cardíaca na mãe. Nesta situação, há que se atentar para o aconselhamento genético, realização de exame para o diagnóstico da cardiopatia fetal no pré-natal e orientações acerca do risco de transmissão da doença materna para o feto em formação(2).

Em alguns casos, a interrupção médica da gestação é indicada em situações em que não se encontram outros meios de manter a vida da gestante, especialmente diante de cardiopatias com risco de morbiletalidade materno-fetal entre 50 e $70 \%{ }^{(7)}$. Na compreensão das mulheres deste estudo, independente da gravidade da cardiopatia e do risco gestacional, referiram pedir ao médico para dar prioridade à vida do bebê e em determinada situação, não interromper a gestação.

De modo semelhante, em revisão integrativa acerca da experiência de mulheres com pré-eclâmpsia, as participantes acreditavam que os bebês não estavam prontos para nascerem e o sentimento de culpa assomou-Ihes diante da necessidade do parto prematuro e da ocorrência de óbito neonatal. Os resultados apontam ainda para o medo da própria morte associado a outros sentimentos negativos ${ }^{(15)}$.

No que diz respeito à dimensão emocional, sabe-se que a ansiedade e estresse devem ser particularmente considerados no âmbito da gestação de alto risco, imprimindo complicações que culminam em piores resultados perinatais e afetando as relações familiares, por alterações do estado de saúde mental ${ }^{(16)}$. A doença cardíaca também está associada a maior desenvolvimento de depressão pós-parto, especialmente diante de complicações maternas. Neste caso, a depressão se manifesta por sentimentos de medo, pânico e tristeza ${ }^{(17)}$.

Sugere-se que intervenções no pré-natal sejam realizadas na perspectiva da prevenção da depressão pós-parto ${ }^{(18)}$. Para tal, torna-se necessário considerar as questões emocionais que envolvem a gestante portadora de cardiopatia, por meio da escuta atentiva durante as consultas e momentos de cuidado à saúde materno-fetal ${ }^{(11)}$.

Em estudo prospectivo que analisou a qualidade de vida durante a gestação e após o parto em mulheres cardiopatas, encontraram-se menores escores nos domínios estado geral de saúde e vitalidade, os quais, ao lado das exigências da maternidade, também podem estar associados com a cardiopatia. Assim como em nossa pesquisa, os diagnósticos mais prevalentes no estudo citado estiveram associados às cardiopatias reumáticas e valvopatias, além de semelhança quanto ao número de gestações, abortos prévios e ausência de planejamento da gravidez mais recente. Quanto a este último aspecto, desperta atenção o resultado de correlação negativa entre aspectos emocionais e ausência de planejamento da gestação após o parto ${ }^{(19)}$.

Assim, a equipe de profissionais de saúde e especialmente a Enfermagem envolvida no período periparto, deve incluir no plano de cuidados assistenciais suporte emocional, encorajamento e avaliações do bem-estar mental das pacientes, face à vulnerabilidade em que se encontram, uma vez que estas questões impactarão fortemente no ciclo gravídico puerperal e na dinâmica familiar após o parto(20).

Neste sentido, tomam-se em paralelo concepções acerca da análise da vulnerabilidade individual, social e programática. Neste plano, aponta-se que a análise da vulnerabilidade individual depende da qualidade da orientação transmitida às pessoas, do conhecimento efetivo que estas incorporam acerca de seus problemas de saúde e da capacidade que detém para transformarem seus cotidianos em busca de modos de vida mais saudáveis ${ }^{(9)}$.

No âmbito social, a vulnerabilidade pode ser entendida como o grau de acesso que as pessoas têm às boas condições de moradia, higiene, lazer, aos bens de consumo e de liberdade para se expressarem. Por fim, na instância programática, a análise da vulnerabilidade é realizada com base nos programas, estratégias e ações governamentais intencionados em dirimir os problemas populacionais em um contexto pluridimensional ${ }^{(9)}$.

No âmbito individual, embora as participantes deste estudo expressassem certo grau de conhecimento acerca do risco de parir devido à doença cardíaca, permaneceram com dificuldades para incorporar os conhecimentos transmitidos ao seu cotidiano, dado o não planejamento da gestação e a permanência de dúvidas, incertezas e temor. Tornaram-se então mais vulneráveis na perspectiva de 
transformação efetiva da sua realidade a partir de si mesmas.

No programático, a vulnerabilidade presentificou-se face às estatísticas que revelam taxas elevadas e crescentes de morbimortalidade materno-fetal por doenças cardíacas ${ }^{(4,6)}$. Apesar da interface do Plano de Ações para enfrentamento das doenças crônicas não transmissíveis, junto à Rede Cegonha no acompanhamento da nutrição, controle de hipertensão e glicemia da gestante ${ }^{(21)}$, estes procedimentos se mostram tecnicamente insuficientes, reducionistas e limitantes, sendo pouco democráticos diante dos recursos que as mulheres necessitam para não se exporem a maiores agravos, bem como seus filhos, que são acompanhados por apenas dois anos após o parto ${ }^{(22)}$.

Em vista disso, ainda que a instituição referência para o risco materno receba e acompanhe a gestante, assegure consultas com diversos profissionais e em alguns casos absorva a mulher e seu filho em um continuum de cuidados após o ciclo gravídico puerperal, não se pode garantir que outras mulheres e crianças estarão sob a mesma vigilância e proteção, o que implica pensar sobre a vulnerabilidade social a que estão expostas.

Aponta-se como limitação advinda do método qualitativo a não generalização dos resultados, porém considera-se a relevância do referencial teórico-metodológico na possibilidade de apontar direcionamentos que podem ser incluídos na prática clínica, de modo a contemplar aspectos ônticos e ontológicos, superando modelos centrados na patologia e não no ser em situação de doença.

\section{- CONSIDERAÇÕES FINAIS}

A análise do movimento existencial da mulher portadora de cardiopatia no vivido do parto apontou em direção ao desvelamento do temor frente ao medo expresso de morrer no parto. Foi possível discutir a vulnerabilidade que cardiopatas vivenciam ao engravidarem. Isto porque, para além das possíveis complicações advindas da doença cardíaca que despertaram medo, ansiedade e estresse nas participantes, residem subjetividades que são apartadas do processo de cuidado em saúde, especialmente no que diz respeito à falta de diálogo e tomada de decisão unilateral acerca do tipo, momento e via de parto.

Ao ampliar esta discussão, acredita-se contribuir para a reflexão de enfermeiros e demais profissionais de saúde acerca da integralidade que deve viger como princípio norteador das práticas assistenciais acolhedoras, individualizadas e intersubjetivas junto a mulheres com gestações de alto risco.

Neste âmbito, deve-se atentar para a escuta ativa, atentiva e perspectiva de comunicação que favoreça o entendimento e enfrentamento de sentimentos negativos da mulher, o que possibilita melhores resultados maternos e neonatais.

\section{REFERÊNCIAS}

1. da Silva RM, de Araújo KNC, Bastos LAC, Moura ERJ. Planejamento familiar: significado para mulheres em idade reprodutiva. Ciênc. saúde coletiva. [Internet] 2011;16(5) [acesso em 18 out 2016]. Disponível: http://dx.doi. org/10.1590/S1413-81232011000500010.

2. Jorge HMF, Hipólito MCV, Masson VA, da Silva RM. Prenatal care and public policies for women's health: integrative review. Rev Bras Promoç Saúde. [Internet] 2015;28(1) [acesso em 18 out 2016]. Disponível: http:// dx.doi.org/10.5020/18061230.2015.p140.

3. Souza JP. A mortalidade materna e os novos objetivos do desenvolvimento sustentável (2016-2030). Rev. Bras. Ginecol. Obstet. [Internet] 2015;37(12) [acesso em 05 nov 2016]. Disponível: http://dx.doi.org/10.1590/SO100720320150005526 .

4. Lage EM, Barbosa AS. Cardiopatias e gravidez. Femina. 2012;40(1):43-50.

5. Kassebaum NJ, Bertozzi-Villa A, Coggeshall MS, Shackelford KA, Steiner C, Heuton KR, et al. Global, regional and national levels and causes of maternal mortality during 1990-2013: a systematic analysis for the Global Burden of Disease Study 2013. The Lancet. [Internet] 2014;384(9947) [acesso em 05 nov 2016]. Disponível: http://dx.doi. 
org/10.1016/S0140-6736(14)60696-6.

6. Reis ZSN, Lage EM, Aguiar RALP, Gaspar JS, Vitral GLN, Machado EG. Associação entre risco gestacional e tipo de parto com as repercussões maternas e neonatais. Rev. Bras. Ginecol. Obstet. [Internet] 2014;36(2) [acesso em 05 nov 2016]. Disponível: http://www.scielo.br/pdf/rbgo/v36n2/0100-7203-rbgo-36-02-00065.pdf.

7. Ministério da Saúde (BR). Departamento de Ações Programáticas e Estratégicas. Gestação de Alto Risco: manual técnico. Brasília: Ministério da Saúde; 2012.

8. de Souza NL, de Araújo ACPF, Costa ICC. Representações sociais de puérperas sobre as síndromes hipertensivas da gravidez e nascimento prematuro. Rev. Latino-Am. Enfermagem. [Internet] 2013;21(3) [acesso em 12 nov 2016]. Disponível: http://dx.doi.org/10.1590/S0104-11692013000300011.

9. Ayres JRCM. O conceito de vulnerabilidade e as práticas de saúde: novas perspectivas e desafios. In: Czeresnia D, Freitas CM, organizadores. Promoção da saúde: conceitos, reflexões, tendências. Rio de Janeiro: Fiocruz; 2003. p.117-39.

10. Avila WS, de Figueiredo MR, Bortolotto L. Gestação em cardiopatias congênitas: visão do cardiologista e do obstetra. Rev Soc Cardiol Estado de São Paulo. 2015;25(3):147-55.

11. Amorim TV, Souza IEO, Salimena AMO, de Melo MCSC, Paiva ACPC, Moura MAV. The everyday of a heart disease high-risk pregnancy: phenomenological study of care relationships. Esc. Anna Nery. [Internet] 2016;20(4) [acesso em 05 nov 2016]. Disponível: http://dx.doi.org/10.5935/1414-8145.20160091.

12. Heidegger M. Ser e Tempo. Vozes: Petrópolis; 2011.

13. Carvalheira APP, Tonete VLP, Parada CMGL. Sentimentos e percepções de mulheres no ciclo gravídico puerperal que sobreviveram à morbidade materna grave. Rev. Latino-Am. Enfermagem. [Internet] 2010;18(6) [acesso em 12 nov 2016]. Disponível: http://dx.doi.org/10.1590/S0104-11692010000600020.

14. Tedesco RP, Passini Junior R, Cecatti JG, Pacagnella RC, Sousa MH. Estimations of preterm birth rate, associated factors and maternal morbidity from a demographic and health survey in Brazil. Matern Child Health J. [Internet] 2013;17(9) [acesso em 12 out 2016]. Disponível: http://dx.doi.org/10.1007/s10995-012-1177-6.

15. Vaerland IE, Vevatne K, Brinchmann BS. An integrative review of mother's experiences of pre-eclampsia. J Obstet Gynecol Neonatal Nurs. [Internet] 2016;45(3) [acesso em 12 nov 2016]. Disponível: http://dx.doi. org/10.1016/j.jogn.2016.02.006.

16. Fairbrother $\mathrm{N}$, Young AH, Janssen $\mathrm{P}$, Antony MM, Tucker E. Depression and anxiety during the perinatal period. BMC Psychiatry. [Internet] 2015;15(206) [acesso em 12 out 2016]. Disponível: http://dx.doi.org/10.1186/ s12888-015-0526-6.

17. Burgut FT, Bener A, Ghuloum S, Sheikh J. A study of postpartum depression and maternal risk factors in Qatar. J Psychosom Obstet Gynaecol. [Internet] 2013;34(2) [acesso em 12 nov 2016]. Disponível: http://dx.doi.org/10.31 09/0167482X.2013.786036.

18. Clatworthy J. The effectiveness of antenatal intervention to prevent postnatal depression in high-risk women. J Affect Disord. [Internet] 2012;137(1-3) [acesso em 12 nov 2016]. Disponível: http://dx.doi.org/10.1016/j. jad.2011.02.029.

19. Meneguin S, Xavier CL, de Santana DG. Quality of life of cardiac patients during pregnancy and after birth. Acta paul. enferm. [Internet] 2016;29(2) [acesso em 12 nov 2016]. Disponível: http://dx.doi.org/10.1590/19820194201600032.

20. Doyle CS. When delivery expectations change: the role nurses play in reducing parental stress. Nurs Womens Health [Internet] 2011;15(6) [acesso em 12 nov 2016]. Disponível: http://dx.doi.org/10.1111/j.1751-486X.2011.01680.x. 21. Brasil. Ministério da Saúde (MS). Departamento de Análise de situação de saúde. Plano de ações estratégicas para o enfrentamento das doenças crônicas não transmissíveis (DCNT) no Brasil 2011-2022. Brasília: Ministério da Saúde; 2011.

22. Ministério da saúde (BR). Portaria n. 1.459, de 24 de junho de 2011: Institui, no âmbito do Sistema Único de Saúde - SUS - a Rede Cegonha. Diário Oficial da União, [Internet] 24 jun 2011 [acesso em 22 dez 2016]. Disponível: http://bvsms.saude.gov.br/bvs/saudelegis/gm/2011/prt1459_24_06_2011.html. 